\title{
Importance of Diagnostic Imaging Technology in asymptomatic patients and therapeutic imaging approach: Child with ruptured brain AVM
}

\author{
Štrbac Tea ${ }^{1}$, Frkanec Stjepan ${ }^{1}$, Stojić Vana ${ }^{1}$, Ćuk Mario $^{2}$ (Mentor), Barišić Nina ${ }^{2}$, Ozretić David ${ }^{3}$, \\ Chapot Rene ${ }^{4}$ \\ ${ }^{1}$ School of Medicine University of Zagreb, Zagreb, Croatia
}

${ }^{2}$ Department of Pediatrics, University Hospital Centre Zagreb, University of Zagreb, School of Medicine

${ }^{3}$ Department of Diagnostic and Interventional Radiology, University Hospital Center Zagreb, University of Zagreb School of Medicine, Zagreb, Croatia

${ }^{4}$ Department of Intracranial Endovascular Therapy Alfried Krupp Krankenhaus, Essen, Germany

DOI: https://doi.org/10.26800/LV-142-supp5-3

\section{Introduction:}

Brain arteriovenous malformation is a type of high flow vascular malformation shunt in which blood flows from a feeding artery to a draining vein forming a connection called nidus (shunting arterioles/venous loops). They may not become clinically evident for several years. Other possible clinical presentations include headaches, seizures, and hemorrhage, which can be a vital complication.

\section{Case:}

Patient is a female child at age of 10 in good general condition who presented after a mild head trauma. Consequently, she had a headache, stiff neck and vomiting. All of these, led to contusi capitis diagnosis which indicated craniogram, emergency head CT and MR. In general, she is a well-being girl, with no other significant diseases in her or family history. During imaging studies AV-malformation was accidentally found on brain CT scans and proven on both MR and DSA. The AVM is located in ventral right cerebellum and its size is close to $3 \mathrm{~cm}$. The main feeders are a right AICA and the left Pica. Venous drainage occurs via an inferior vermian vein. Another finding is a bleeding occurred on the venous outflow. A treatment is justified due to the young age and the previous bleeding. Surgery is difficult in the base of skull location, radiation therapy is restricted in its efficiency due to the size. It is likely that transarterial embolization may lead to ischemic complication, specifically with regard to the close location of cranial nerves. However, there is no doubt that endovascular treatment can cure the patient by associating transvenous to transarterial embolization.

\section{Conclusion:}

Recent advances in medical imaging technologies such as CT scans, MRIs have a huge impact in modern medicine. When it is combined with the broad knowledge of clinical experts, it may result with better outcome compared to standard methods of diagnosis and treatment.

Keywords: AVM, DSA, embolization, imaging 